7-11-2017

\title{
Ruptured mycotic aneurysms: Report and outcomes of two surgically managed patients
}

\author{
Aleena Khan \\ Aga Khan University \\ Muhammad Waqas \\ Aga Khan University, muhammad.waqas@aku.edu \\ Waseem Mehmood Nizamani \\ Aga Khan University \\ Muhammad Ehsan Bari \\ Aga Khan University, ehsan.bari@aku.edu
}

Follow this and additional works at: https://ecommons.aku.edu/pakistan_fhs_mc_surg_neurosurg

Part of the Neurology Commons, Neurosurgery Commons, and the Surgery Commons

\section{Recommended Citation}

Khan, A., Waqas, M., Nizamani, W. M., Bari, M. E. (2017). Ruptured mycotic aneurysms: Report and outcomes of two surgically managed patients. Surgical Neurology International, 8, 144.

Available at: https://ecommons.aku.edu/pakistan_fhs_mc_surg_neurosurg/143 


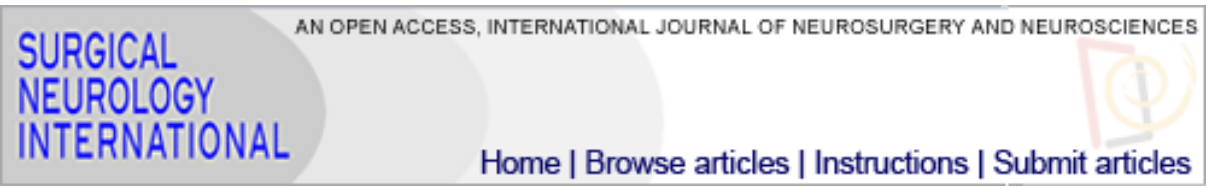

Surg Neurol Int. 2017; 8: 144.

PMCID: PMC5523478

Published online 2017 Jul 11. doi: $\underline{10.4103 / \text { sni.sni } 7817}$

PMID: $\underline{28781921}$

\title{
Ruptured mycotic aneurysms: Report and outcomes of two surgically managed patients
}

\author{
Aleena Khan, Muhammad Waqas, Waseem M. Nizamani, and Muhammad E. Bari ${ }^{*}$ \\ Department of Neurosurgery, Aga Khan University Hospital, Karachi, Pakistan \\ Aleena Khan: aleenakhan93@gmail.com; Muhammad Waqas: shaiq waqas@hotmail.com; Waseem M. Nizamani: \\ dr waseemayub@hotmail.com; Muhammad E. Bari: ehsan.bari@aku.edu \\ Corresponding author
}

Received 2017 Feb 20; Accepted 2017 Apr 21.

Copyright : @ 2017 Surgical Neurology International

This is an open access article distributed under the terms of the Creative Commons Attribution-NonCommercial-ShareAlike 3.0 License, which allows others to remix, tweak, and build upon the work non-commercially, as long as the author is credited and the new creations are licensed under the identical terms.

This article has been cited by other articles in PMC

\section{Background:}

Mycotic aneurysm is a rare potentially life-threatening complication of infective endocarditis (IE). Little data is available on the management and outcomes of ruptured mycotic aneurysms with large intracerebral hematoma. Few cases have been described on the management of mycotic aneurysm in the presence of life-threatening hematoma and mass effect.

\section{Case Description:}

Go to:

We are presenting two cases of ruptured mycotic aneurysm with intracerebral hematoma and impending brain herniation. Both patients had signs of high intracranial pressure and required urgent surgical evacuation of clot. One patient survived while the other patient expired soon after surgery.

\section{Conclusion:}

Mycotic aneurysm of middle cerebral artery (MCA) in IE with intracranial hemorrhage is rare and urgent surgical decompression, and aneurysmal clipping can be lifesaving.

Keywords: Infective endocarditis, intracranial hemorrhage, mycotic aneurysm, neurosurgical emergency, rheumatic heart disease

Intracranial mycotic aneurysms are a rare complication of infective endocarditis (IE) associated with profound morbidity and mortality.[2] Septic emboli from the heart seed to distant vessels, resulting in vascular wall infection, necrosis, and mycotic aneurysm formation. There is a high risk of aneurysmal rupture leading to intracranial hemorrhage. Standard treatment consists of antibiotic therapy combined 
with surgical debridement of necrotic tissue.[2] Ruptured aneurysm may require endovascular coiling or surgical clipping. Few cases have been described on the management of mycotic aneurysm in the presence of life-threatening hematoma and mass effect. We report two cases of IE complicated by ruptured intracerebral mycotic aneurysm with life-threatening mass effect.

\section{CASE DESCRIPTION}

\section{Case 1}

Clinical presentation A 16-year-old female student presented in emergency department (ED) with sudden severe, throbbing right-sided headache followed by an episode of seizure involving jerky movements of left side of her body. Two months earlier, she had been diagnosed with rheumatic heart disease, and was taking antibiotics for IE for 1 month. Although she had not spiked fever in the past 3 days, she had been intermittently febrile for 6 months. Her initial blood cultures had revealed Streptococcus mitis growth.

On examination, she was drowsy, with no eye opening. She was localizing from her right side. Her pupils were equal and reactive. The pulse rate was regular (98 beat per minute) and blood pressure was 122/76 mm Hg. Cardiac auscultation revealed normal first and second heart sounds, a pansystolic murmur in the mitral region and S3 gallop rhythm.

Investigations At the time of admission, her hemoglobin was $8.9 \mathrm{gm} / \mathrm{dL}$ and leukocyte count was elevated $\left(22.7 \times 109 / \mathrm{mm}^{3}\right)$. Erythrocyte sedimentation rate $(\mathrm{ESR})$ and C-reactive protein $(\mathrm{CRP})$ were $102 \mathrm{~mm} / \mathrm{h}$ and $6.2 \mathrm{mg} / \mathrm{dL}$, respectively. Blood cultures were found to grow Streptococcus mitis. Transthoracic echocardiograph showed a severely dilated left atrium, moderate mitral, and tricuspid regurgitation with an echogenic area measuring $12 \times 8 \mathrm{~mm}$ in size located on mitral valve consistent with vegetation. Electroencephalogram showed asymmetric electrical activity with right-sided suppression.

On magnetic resonance imaging (MRI), a large intraparenchymal hematoma was identified in the right temporoparietal region with intraventricular extension, mass effect, and midline shift. A small enhancing focus was seen in M2 segment of right middle cerebral artery (MCA) likely representing mycotic aneurysm [표마re 1] . 


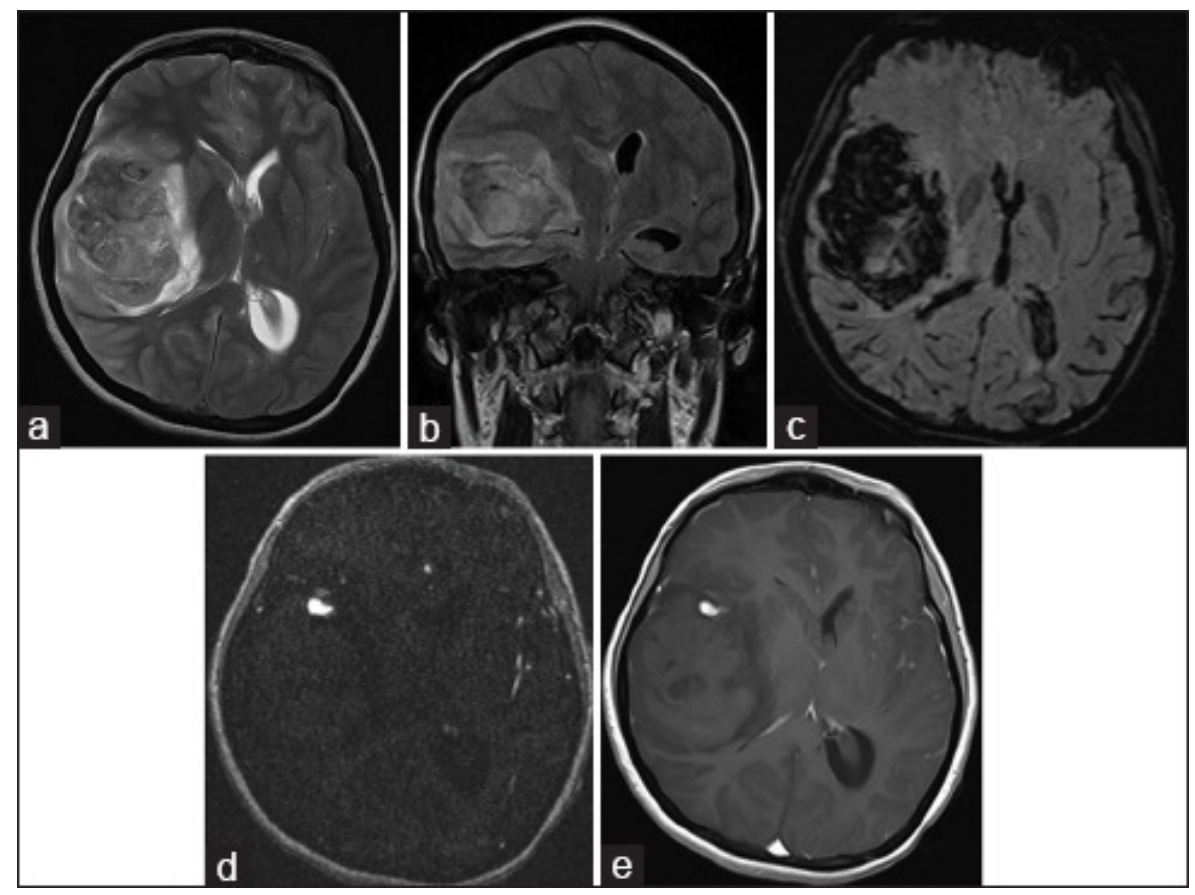

\section{Figure 1}

(a-e) Axial T2WI and coronal FLAIR shows right sided intraparenchymal hematoma causing mass effect and midline shift. (c) Signal dropout is best demonstrated on axial SWI. (e and d) Post contrast T1WI and MRA axial images shows right MCA mycotic aneurysm

Treatment Due to a sudden decrease in responsiveness soon after arrival, she was sedated, intubated, and admitted to the intensive care unit (ICU). She was started on meropenem (1 gram every 8 hours) and vancomycin (1 gram every 12 hours). Over few hours her neurological status got worse. Pupils became anisocoric and motor response dropped to bilateral abnormal extension. Family after much delay consented for surgery. A right frontotemporal craniotomy was performed for clot evacuation. As soon as the duratomy was performed brain tissue started herniating out of bone defect. Normal parenchyma of frontal and temporal lobe had to be resected along with clot evacuation to enable wound closure. A fusiform aneurysm was noticed in M3 part of MCA which we left unclipped as the patient did not seem salvageable. Wound was closed rapidly without bone flap. She was shifted to ICU where she did not show any improvement. Family was explained poor prognosis and ventilator support was withdrawn gradually.

Outcome and follow-up Patient expired over next 24 hours.

\section{Case 2}

Clinical presentation A 30-year-old male presented in ED with right hemiparesis and slurred speech for 1 day. He had been intermittently febrile for the past 5-6 months and was diagnosed with IE 6 weeks prior to presentation, for which he was receiving intravenous antibiotics.

On examination, he was afebrile. His heart rate was 100 beats per minute (bpm) and blood pressure was 100/50 mm Hg. He had mild clubbing (Grade 1), with an otherwise unremarkable general examination. He was drowsy, disoriented, and unable to follow commands. He had eye opening to command and localized from his left side. 
Initial blood work up showed hemoglobin of $11.6 \mathrm{gm} / \mathrm{dL}$. Leukocyte count was elevated (i.e., $13.9 \times$ $109 / \mathrm{mm}^{3}$ ). Blood cultures did not grow any organism. ESR was 79 . Transthoracic echocardiography showed vegetations on the aortic valve measuring $22 \mathrm{~mm} \times 13 \mathrm{~mm}$ and mitral valve measuring $20 \mathrm{~mm}$ $\times 11 \mathrm{~mm}$. Preliminary MRI suggested acute hemorrhagic infarction of left MCA with significant cerebral edema, midline shift, and mass effect. CT angiogram of brain exhibited a fusiform aneurysm of posterior sylvian branch of left MCA [iigure 2].

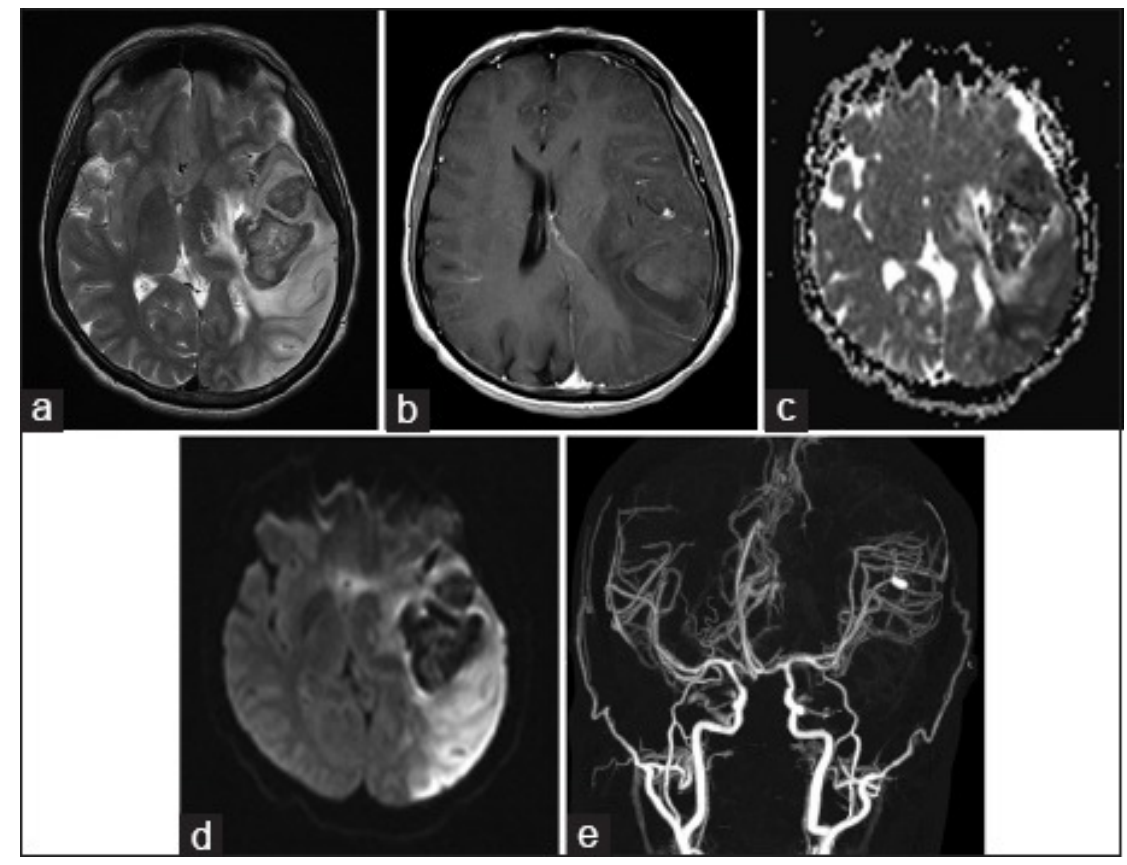

\section{Figure 2}

(a-e) Axial T2WI \& DWI shows large left sided intraparenchymal hematoma with left MCA territory infarction. (b and e) Post contrast T1WI and MIP CT angiogram shows left MCA aneurysm

Treatment Initially, a conservative approach was employed with administration of antibiotics (meropenem 1 gram every 8 hours and vancomycin 1 g every 12 hours). On second day of admission we observed a drop in motor and verbal response. Glasgow coma scale dropped from 10 to 8 . He was rushed to theatre immediately where frontotemporal decompressive craniectomy was done, clot evacuated and aneurysm of M3 clipped. Postoperatively he was shifted to ICU where he started showing gradual improvement. Over a week he started obeying commands and started moving left side. Cranioplasty was performed after 3 months. He was managed with a multidisciplinary approach with cardiothoracic surgery and infectious disease department.

Outcome and follow-up Good recovery with mild residual disability was noted at 6 months follow-up.

Symptomatic neurological manifestations are seen in 35\% of IE patients.[9] Septic emboli are known to dislodge from heart and occlude vasa vasorum of intracerebral vasculature, extending infection through intima. Subsequent necrosis of the adventitia and media, and dilation of vessel wall causes mycotic aneurysmal formation. 
We have described two cases of ruptured mycotic aneurysm with large intracerebral hematomas causing immediate threat to life. A review of literature revealed that rupture rates vary from $<2 \%$ to $72 \% .[\underline{2}, \underline{5}, \underline{6}, \underline{8}]$ Due to impending herniation both patients underwent surgery with different outcomes. The main purpose of surgery was temporal decompression and clot evacuation to prevent brain stem compression. In case 2, patient survived the acute effects of hematoma because of early intervention. The other patient presented in a poor neurological condition. The intervention was delayed for several hours because of delay in MRI and then failure of family to arrive at an early decision. We believe that a CT scan instead of an MRI was the more appropriate investigation for patient in case 1. Mortality rates have been reported up to $30 \%$ for unruptured and $80 \%$ for ruptured mycotic aneurysms.[2, $, \underline{4}, \underline{5}]$ Several authors have recommended cerebral angiogram in patients presenting with any neurological deficit.

These cases are unique in several respects. Allen et al. have presented the largest case series of mycotic aneurysms so far with 26 patients.[1] Of them $60 \%$ had rupture but only five patients required clipping of aneurysm. Only eight patients had intraparenchymal hemorrhage.[1] The series did not elaborate upon urgent need of surgery and clot evacuation in any of these cases.

There are several other case reports of mycotic aneurysms, but very few have described ruptured mycotic aneurysm with impending herniation.

In a series by Kannoth et al. 10 patients had mycotic aneurysm due to IE.[్] In his cohort of 25 mycotic aneurysm patients five had early surgery yet no case of emergent surgical procedure for decompression and aneurysmal repair was elaborated in the series.[]ㅡ

Mycotic aneurysms rarely present with subdural hematoma as seen in our case 2 . Only 10 such cases have been reported.[ㄹ] It must therefore be highlighted that the presence of subdural hematoma does not preclude the need of angiogram in patients with history of endocarditis.

Antibiotics are the mainstay of treatment of unruptured mycotic aneurysms. Literature supports a minimum at 4-6 weeks of antibiotic therapy with serial CT scans and angiography to follow the status of the mycotic aneurysm.[ㄹ,, ] Ruptured aneurysms warrant surgical approach with clipping, endovascular coiling, or aneurysmal trapping, with urgent decompression for those with hematoma and significant mass effect.

Mycotic aneurysm of MCA in the setting of IE with intraparenchymal hemorrhage and/or subdural hematoma is rare. An urgent angiogram is warranted in any IE patient presenting with neurological symptoms. If performed timely urgent surgical decompression and aneurysmal clipping can be lifesaving for cases with impending herniation.

\section{Financial support and sponsorship}

Nil.

\section{Conflicts of interest}

There are no conflicts of interest. 
1. Allen L, Fowler A, Walker C, Derdeyn C, Nguyen B, Hasso A, et al. Retrospective review of cerebral mycotic aneurysms in 26 patients: Focus on treatment in strongly immunocompromised patients with a brief literature review. Am J Neuroradiol. 2013;34:823-7. [ubMed]

2. Ducruet AF, Hickman ZL, Zacharia BE, Narula R, Grobelny BT, Gorski J, et al. Intracranial infectious aneurysms: A comprehensive review. Neurosurg Rev. 2010;33:37-46. [PubMed]

3. Frazee JG, Cahan LD, Winter J. Bacterial intracranial aneurysms. J Neurosurg. 1980;53:633-41. [PubMed]

4. Huang J, McGirt MJ, Gailloud P, Tamargo RJ. Intracranial aneurysms in the pediatric population: Case series and literature review. Surg Neurol. 2005;63:424-32. [ubMed]

5. Kannoth S, Thomas SV. Intracranial microbial aneurysm (infectious aneurysm): Current options for diagnosis and management. Neurocrit Care. 2009;11:120-9. [PubMed]

6. Kundra SN. Management of intracranial infectious aneurysms: A series of 16 cases. Neurosurgery. 2003;53:245-6. [ubMed]

7. Peters PJ, Harrison T, Lennox JL. A dangerous dilemma: Management of infectious intracranial aneurysms complicating endocarditis. Lancet Infect Dis. 2006;6:742-8. [PubMed]

8. Phuong LK, Link M, Wijdicks E. Management of intracranial infectious aneurysms: A series of 16 cases. Neurosurgery. 2002;51:1145-52. [PubMed]

9. Snygg-Martin U, Gustafsson L, Rosengren L, Alsiö Å, Ackerholm P, Andersson R, et al. Cerebrovascular complications in patients with left-sided infective endocarditis are common: A prospective study using magnetic resonance imaging and neurochemical brain damage markers. Clin Infect Dis. 2008;47:23-30. [PubMed]

Articles from Surgical Neurology International are provided here courtesy of Wolters Kluwer -- Medknow Publications 